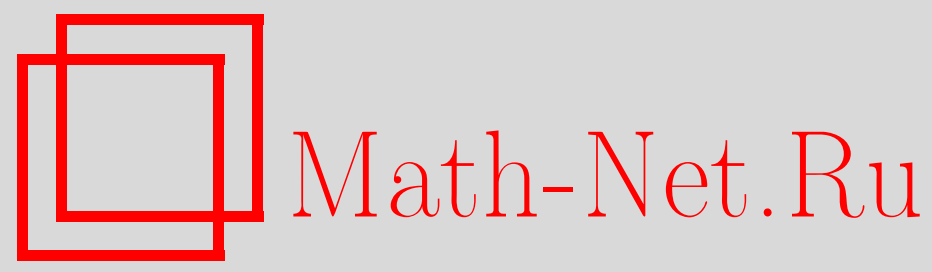

А. И. Кожанов, Начально-краевая задача для уравнений типа обобщенного уравнения Буссинеска с нелинейным источником, Матем. заметки, 1999, том 65, выпуск 1, 70-75

DOI: https://doi.org/10.4213/mzm1029

Использование Общероссийского математического портала Math-Net.Ru подразумевает, что вы прочитали и согласны с пользовательским соглашением http://www.mathnet.ru/rus/agreement

Параметры загрузки:

IP : 54.164 .48 .24

26 апреля 2023 г., 15:06:29

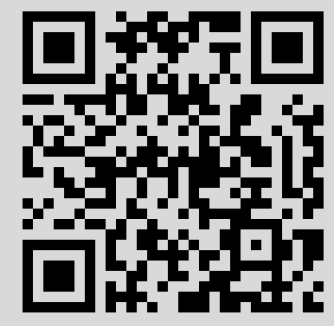




\section{НАЧАЛЬНО-КРАЕВАЯ ЗАДАЧА ДЛЯ УРАВНЕНИЙ ТИПА ОБОБЩЕННОГО УРАВНЕНИЯ БУССИНЕСКА С НЕЛИНЕЙНЫМ ИСТОЧНИКОМ}

\section{А. И. Кожанов}

Для обобщенного уравнения Буссинеска с нелинейньм источником

$$
u_{t}-\Delta \psi(u)-\Delta u_{t}+q(u)=0
$$

доказывается принцип сравнения для решений первой начально-краевой задачи. С помощью принципа сравнения в случае степенных функций $\psi(\xi)$ и $q(\xi)$ доказываются новые теоремы существования и несушествования решений первой начально-краевой задачи.

Библиограффия: 9 названий.

Обобщенное уравнение Буссинеска как математический объект и как объект математического моделирования фильтрационных процессов исследовалось в работах [1]-[3] и др. В настояшей работе будет изучаться обобщенное уравнение Буссинеска с нелинейным неподчиненным немонотонньм источником, а именно, уравнение

$$
u_{t}-\Delta \psi(u)-\Delta u_{t}+q(u)=0
$$

Основным инструментом исследования при этом будет доказанный ниже принпип сравнения. Идеи, связанные с приншипом сравнения, для уравнений вида $(1)$ использовались ранее в работах [2], [4]-[6]. В работе [2] рассматривался случай $q(\xi) \equiv 0$; для первой начально-краевой задачи были построены ограниченные на любом конечном промежутке изменения переменной $t$ (но, возможно, растущие при $t \rightarrow \infty$ ) верхние решения и, тем самьм, было доказано существование регулярных решений поставленной задачи в цилиндре конечной высоты с ограниченной областью в основании. В работе [4], принадлежащей автору, рассматривались более общие, чем (1), уравнения, однако, принцип сравнения использовался лишш для доказательства неотрицательности решений, в остальном же для доказательства сушествования регулярных решений первой начально-краевой задачи (также в цилиндре конечной высоты и с ограниченным основанием) использовались идеи, связанные с интегральньми априорньми оценками, теоремами вложения и условиями подчинения. В работах [5], [6], также принадлежащих автору, рассматривался случай $\psi(\xi) \equiv \xi, q(\xi) \sim \xi^{p}, p>1$, и были построены убывающие при

Работа выполнена при финансовой поддержке Российского фонда фундаментальных исследований, грант № 96-01-01597. 
$t \rightarrow \infty$ верхние решения и неограниченные на конечном промежутке изменения переменной нижние решения; с помошью построенных верхних и нижних решений были доказаны теоремы существования и несуществования глобальных решений задачи Коши и начально-краевых задач.

Именно к методике работ [5], [6] и будет близка методика, применяемая в настояшей работе.

Перейдем к содержательной части работы. Пусть $x$ есть точка ограниченной области $D$ пространства $\mathbb{R}^{n}, \Gamma=\partial D, Q=D \times(0, T)$. В цилиндре $Q$ рассмотрим следующую начально-краевую задачу: найти решение уравнения (1) в $Q$, удовлетворяющее условиям

$$
u(x, 0)=u_{0}(x), \quad x \in D,\left.\quad u\right|_{\Gamma \times(0, T)}=\mu_{0}(x, t), \quad x \in \Gamma, \quad t \in(0, T) .
$$

ТЕорема 1 (приншип сравнения). Пусть $и(x, t)$ есть регулярное решение краевой задачи (1), (2), v(x,t) есть решение неравенства

$$
v_{t}-\Delta \psi(v)-\Delta v_{t}+q(v)>0(<0)
$$

в иилиндре $Q$, удовлетворяющее условиям

$$
v(x, 0)=v_{0}(x), \quad x \in D,\left.\quad v\right|_{\Gamma \times(0, T)}=\mu_{1}(x, t), \quad x \in \Gamma, \quad t \in(0, T) .
$$

Если функиия $\lambda(\xi)=q(\xi)-\psi(\xi)$ есть убивающая по $\xi$ при $\xi \in \mathbb{R}$ функиия и если при $x \in \bar{D}$ выполняется неравенство $v_{0}(x)>u_{0}(x)$ (соответственно $v_{0}(x)<u_{0}(x)$ ), а при $x \in \Gamma, t \geqslant 0$ выполняются неравенства

$$
\mu_{1 t}(x, t)+\psi\left(\mu_{1}(x, t)\right)>\mu_{0 t}(x, t)+\psi\left(\mu_{0}(x, t)\right), \quad \mu_{1}(x, t)>\mu_{0}(x, t)
$$

(соответственно

$$
\left.\mu_{1 t}(x, t)+\psi\left(\mu_{1}(x, t)\right)<\mu_{0 t}(x, t)+\psi\left(\mu_{0}(x, t)\right), \quad \mu_{1}(x, t)<\mu_{0}(x, t)\right)
$$

то для $x \in \bar{D} u t \geqslant 0$ справедливо неравенство $v(x, t)>u(x, t)$ (соответственно $v(x, t)<u(x, t))$.

ДокАЗАТЕЛЬСТво. Пусть выполнена первая из указанных в условии теоремы группа неравенств. Воспользуемся идеей доказательства принципа неотрицательности решения работы [4].

Пусть неравенство $v(x, t)>u(x, t)$ всюду в цилиндре $Q$ не выполняется, и пусть число $t_{0}$ такое, что $t_{0}>0$; при $t \in\left(0, t_{0}\right), x \in D$ вьполняется неравенство $v(x, t)>u(x, t)$ и найдется точка $x_{0} \in D$ такая, что вьполняется $v\left(x_{0}, t_{0}\right)=u\left(x_{0}, t_{0}\right)$. Обозначим $w=v_{t}-u_{t}+\psi(v)-\psi(u)$. Для функции $w$ при $x \in D, t \in\left[0, t_{0}\right]$ справедливы неравенства

$$
\Delta w-w<(q(v)-\psi(v))-(q(u)-\psi(u)) \leqslant 0 .
$$

Кроме того, условия теоремы означают, что при $x \in$ Ги любом фиксированном $t \in\left[0, t_{0}\right]$ справедливо неравенство $w(x, t)>0$. Вследствие принципа максимума для эллиптических уравнений имеем, что последнее неравенство будет вьполняться при $x \in \bar{D}$ и всех $t \in\left[0, t_{0}\right]$. В частности, в точке $\left(x_{0}, t_{0}\right)$ будет вьполняться неравенство $v_{t}\left(x_{0}, t_{0}\right)-$ $u_{t}\left(x_{0}, t_{0}\right)>0$. Но тогда найдется число $t^{*}$ такое, что $0 \leqslant t^{*}<t_{0}$ и при $t \in\left(t^{*}, t_{0}\right]$ будет 
справедливо неравенство $v_{t}\left(x_{0}, t\right)-u_{t}\left(x_{0}, t_{t}\right)>0$. Интегрируя последнее неравенство, получаем

$$
v\left(x_{0}, t_{0}\right)-u\left(x_{0}, t_{0}\right)>v\left(x_{0}, t\right)-u\left(x_{0}, t\right)>0,
$$

что противоречит предположению о совпадении $v\left(x_{0}, t_{0}\right)$ и $u\left(x_{0}, t_{0}\right)$.

Полученное противоречие опровергает предположение о невыполнении неравенства $v(x, t)>u(x, t)$ всюду в $Q$.

Аналогично анализируется случай, когда вьполняется вторая группа неравенств.

Теорема доказана.

В соответствии с [7] функция $v(x, t)$, удовлетворяющая первым неравенствам из условия теоремы, назьвается верхним решением задачи $(1),(2)$, функция $v(x, t)$, удовлетворяющая вторым неравенствам, - нижним. Как известно, для доказательства сушествования или несуществования глобальных решений задачи $(1),(2)$ необходимо построить подходяшие верхние и нижние решения.

Заметим, что условие на функцию $\lambda(\xi)$ можно ослабить.

Пусть $v_{+}(x, t), v_{-}(x, t)$ есть соответствуюшие верхнее и нижнее решения. Обозначим

$$
a=\inf _{x \in \bar{D}, t \geqslant 0} v_{-}(x, t), \quad b=\sup _{x \in \bar{D}, t \geqslant 0} v_{+}(x, t) .
$$

Имеет место

СлЕДСТВИЕ. Пусть а и $b$ - конечные числа, функиия $\lambda(\xi)$ является убьвающей функиией на $[a, b]$ и допускает непрерывное продолэсене на всю числовую ось $c$ сохранением убывания. Тогда если выполнены условия теоремы 1, то регулярное решение задачи (1), (2) будет удовлетворять неравенствам $v_{-}(x, t)<u(x, t)<$ $v_{+}(x, t)$ для всех $x \in \bar{D}$ и всех $t \geqslant 0$.

Доказательство следствия очевидно.

Ниже в работе будем в основном изучать случай степенных функций $\xi(\psi)$ и $q(\xi)$, поскольку именно такие функции часто встречаются при моделировании реальных физических процессов [1], [2], [7].

Поскольку область $D$ ограничена, ее можно заключить в некоторьй шар с центром в начале координат; пусть $R$ есть радиус минимального из таких шаров.

ТЕОРЕМА 2. Пусть в уравнении (1) выполняется

$$
\psi(\xi) \equiv \xi^{m}, \quad q(\xi) \equiv-\beta \xi^{p}, \quad p \geqslant m \geqslant 2, \quad \beta \geqslant 0,
$$

и пусть $\mu_{0}(x, t) \equiv 0$, функиия $u_{0}(x)$ неотрицательна, принадлежст классу $C^{2}(\bar{D})$ и удовлетворяет условию согласования, а граница области $D$ принадлежит классу $C^{2+\varkappa}, \varkappa>0$. Тогда если положительные постоянные $A, T_{0}, \alpha$ и $\beta$ таковы, что выполняются неравенства

$$
\begin{gathered}
(m-1) \alpha^{m-1} A^{m-1}>1, \quad u_{0}(x)<A T_{0}^{-1 /(m-1)}\left(\alpha+|x|^{2}\right), \\
\frac{2 n}{m-1}+4 m(m-1) \alpha^{m-1} A^{m-1}> \\
\frac{R_{0}}{m-1}+4 m(m-1) A^{m-1} R_{0}^{m-1} \\
+2 m n A^{m-1} R_{0}^{m-1}+\beta A^{p-1} R_{0}^{p},
\end{gathered}
$$

әде $R_{0}=\alpha+R$, то задача (1), (2) будет иметь регулярное решение, и это решение будет удовлетворять неравенствам

$$
0 \leqslant u(x, t) \leqslant R_{0} A\left(T_{0}+t\right)^{-1 /(m-1)}
$$

для всех $t \geqslant 0$. 
ДокАЗАТЕЛЬСТво. Условия теоремы таковы, что функция

$$
v(x, t)=A\left(T_{0}+t\right)^{-1 /(m-1)}\left(\alpha+|x|^{2}\right)
$$

удовлетворяет неравенствам

$$
v_{t}+v^{m}>0, \quad v_{t}-\Delta v^{m}-\Delta v_{t}-\beta v^{p}>0
$$

Следовательно, согласно условиям, связьвающим функцию $v(x, t)$, начальную функцию $u_{0}(x)$ и граничную функцию $\mu_{0}(x, t)$, и теореме 1 регулярное решениезадачи $(1),(2)$ будет ограничено сверху функцией $v(x, t)$. Кроме того, решение задачи $(1),(2)$ будет неотрицательным [2], [4]. Применяя теперь метод срезок [8], [9], нетрудно стандартным образом доказать существование регулярного решения задачи $(1),(2)$; как уже было сказано выше, данное решение будет удовлетворять требуемым неравенствам.

Теорема доказана.

Заметим, что завершение доказательства теоремы 2 можно было бы провести и методом работы [2]. Как уже говорилось вьше, случай $m=2, \beta=0$ (т.е. случай собственно обобщенного уравнения Буссинеска) рассмотрен в работе [2], в которой доказано существование возможно растушего при $t \rightarrow+\infty$ решения первой начально-краевой задачи; теорема 2 говорит о существовании убывающего степенным образом при $t \rightarrow+\infty$ решения. И еще заметим, что случай $m=1$ рассмотрен автором в работах [5], [6].

ТЕОРема 3. Пусть в уравнении (1) выполняется

$$
\psi(\xi) \equiv \xi^{m}, \quad q(\xi) \equiv \beta \xi^{p}, \quad p \geqslant m \geqslant 2, \quad \beta>0,
$$

и пусть р и $\mathrm{m}$ - натуральные числа, $\mu_{0}(x, t) \equiv 0$, функиия $u_{0}(x)$ неотричательна, принадлежит классу $C^{2}(\bar{D})$ и удовлетворяет условию согласования, а граница области $D$ принадлежит классу $C^{2+\varkappa}, \varkappa>0$. Тогда если для положстельных постоянных $A, T_{0}, \alpha$ и выполняются неравенства из условия теоремы 1 и если дополнительно выполняется

$$
A T_{0}^{-1 /(m-1)}\left(\alpha+R^{2}\right)<\left(\frac{m}{p \beta}\right)^{1 /(p-m)}
$$

при $p>m, \beta<1$ при $p=m$, то задача (1), (2) будет иметь регулярное решение, и это решение будет удовлетворять неравенствам

$$
0 \leqslant u(x, t) \leqslant R_{0} A\left(T_{0}+t\right)^{-1 /(m-1)}
$$

для всех $t \geqslant 0$.

ДокАЗАТЕЛЬСтво. Рассмотрим случай $p>m$. Заметим, что функция $q(\xi)-\psi(\xi)$ убывает на интервале $\left(0,(m /(p \beta))^{1 /(p-m)}\right)$. Пусть $\widetilde{\psi}(\xi)$ и $\widetilde{q}(\xi)$ - функции, совпадающие соответственно с $\psi(\xi)$ и $q(\xi)$ при $\xi \in\left[0,(m /(p \beta))^{1 /(p-m)}\right]$ и такие, что

$$
\widetilde{\psi}(\xi)=\left\{\begin{aligned}
\xi^{m} & \text { при } \xi<0, \text { если } m \text { нечетно, } \\
-\xi^{m} & \text { при } \xi<0, \text { если } m \text { четно, }
\end{aligned} \quad \widetilde{q}(\xi) \equiv 0 \text { при } \xi<0,\right.
$$

$\widetilde{\psi}(\xi) \in C^{2}(\mathbb{R}), \widetilde{q}(\xi) \in C(\mathbb{R}), \widetilde{q}(\xi)-\widetilde{\psi}(\xi)$ есть убьвающая функция при $\xi>(m /(p \beta))^{1 /(p-m)}$. 
Нетрудно проверить, что функция $v_{-}(x, t)=-\varepsilon \exp (t), \varepsilon>0$, является нижним решением для уравнения

$$
u_{t}-\Delta \widetilde{\psi}(u)-\Delta u_{t}+\widetilde{q}(u)=0
$$

при вьполнении начальных и краевьх условий $(2)$ с $\mu_{0}(x, t) \equiv 0$. В силу условий теоремы функция $v_{+}(x, t)=A\left(T_{0}+t\right)^{-1 /(m-1)}\left(\alpha+|x|^{2}\right)$ будет верхним решением для того же уравнения при тех же начальных и краевых условиях. Согласно теореме 1 решение уравнения (3) будет удовлетворять неравенствам

$$
-\varepsilon e^{t}<u(x, t)<A\left(T_{0}+t\right)^{-1 /(m-1)}\left(\alpha+|x|^{2}\right) .
$$

Поскольку $\varepsilon$ есть число произвольное, отсюда следует, что решение уравнения (3), удовлетворяющее условиям $(2)$ с $\mu_{0}(x, t) \equiv 0$, будет неотрицательньм. Но тогда такое решение будет решением уравнения (1).

Применяя теперь вновь метод срезок или метод работы [2], нетрудно завершить доказательство теоремы в случае $p>m$.

Случай $p=m$ анализируется в целом аналогично, но более просто, поскольку при $\beta<1$ функция $\lambda(\xi)=(\beta-1) \xi^{m}$ будет убывающей при $\xi>0$.

Теорема доказана.

Tеорема 4. Пусть область $D$ eсmь uар $D=\left\{x \in \mathbb{R}^{n}:|x|<R\right\}, \psi(\xi)$ u $q(\xi)$ имеют тот же вид, что и в теореме 2 , но с дополнительным условием $p>m$. Тогда если граничная функиия $\mu_{0}(x, t)$ и начальная функция $u_{0}(x)$ задачи $(1),(2)$ есть гладкие функиии такие, что для положительных постоянных $A, T_{0}$ и $\gamma$ выполняются неравенства

$$
\begin{gathered}
\mu_{0 t}+\left.\mu_{0}^{m}\right|_{|x|=R}>-A \lambda\left(\gamma R^{2}\right)^{\lambda-1}+A^{m}\left(\gamma R^{2}\right)^{\lambda m}, \\
\left.\mu_{0}\right|_{|x|=R}>A\left(\gamma R^{2}\right)^{\lambda}, \quad u_{0}(x)>A\left(T_{0}+\gamma|x|^{2}\right)^{\lambda}, \\
\left(T_{0}+\gamma R^{2}\right)^{\lambda_{1}}+2 A^{m-1} \gamma m(n-2 m)+4 A^{m-1} \gamma m^{2} \lambda \leqslant 0, \\
2 \gamma \lambda(\lambda-1)\left(T_{0}+\gamma R^{2}\right)^{\lambda_{1}}+4 T_{0} A^{m-1} \gamma \lambda m(\lambda m-1)<A^{p-1},
\end{gathered}
$$

где $\lambda=-2 /(p-m), \lambda_{1}=2(m-1) /(p-m)$, то гладкое решение задачи $(1),(2)$ будет таким, что $u(0, t) \rightarrow+\infty$ при $t \rightarrow T_{0}-0$.

ДокАЗАТЕЛЬСтво. Для функции $v(x, t)=A\left(T_{0}-t+\gamma|x|^{2}\right)$ в цилиндре $Q_{T_{0}}=D \times$ $\left(0, T_{0}\right)$ вьполнены все условия теоремы 1 , необходимые для того, чтобы $v(x, t)$ была нижним решением задачи (1), (2). Тогда для гладкого решения задачи $(1),(2)$, если оно существует, обязано выполняться неравенство $u(x, t)>v(x, t)$ при $x \in D, t \in\left(0, T_{0}\right)$. Отсюда и получаем требуемое утверждение.

Теорема доказана.

Теорема 4 означает, что ограниченная при $t \in\left[0, T_{0}\right]$, хотя и растущая, граничная функция $\mu_{0}(x, t)$ может индуцировать "режим с обострением" (другими словами, особенность приносится сверху).

В завершение работы приведем один обший результат о несуществовании глобальных решений.

Пусть $z_{1}$ - первое собственное число однородной задачи Дирихле для оператора Лапласа в области $D, w_{1}(x)$ - первая собственная функция. Обозначим $Q(\xi)=z_{1} \psi(\xi)-q(\xi)$. 
ТЕоремА 5. Пусть начальная функиия $u_{0}(x)$ такова, что

$$
\int_{D} u_{0}(x) w_{1}(x) d x \geqslant \xi_{0}>0
$$

и пусть при $\xi \geqslant \xi_{0}$ выполняется $Q(\xi)>0, Q^{\prime \prime}(\xi) \geqslant 0$. Кроме того, пусть выполняется

$$
\int_{\xi_{0}}^{\infty} \frac{d \xi}{Q(\xi)}<+\infty
$$

Тогда для любого неотрицательного регулярного решения (1), (2) будет выполнятвся

$$
\varlimsup_{t \rightarrow T^{*}-0} \sup _{x \in \bar{D}} u(x, t)=+\infty
$$

для некоторого конечного числа $T^{*}$.

Доказательство теоремы 5 проводится полностью аналогично доказательству такого же утверждения для параболических уравнений с нелинейным источником (см. [7]).

\section{СПИСОК ЦИТИРОВАННОЙ ЛИТЕРАТУРЫ}

[1] Дзеккер Е. С. Обобщение уравнения движения грунтовых вод со свободной поверхностью // Докл. АН СССР. 1972. Т. 202. № 5. С. 1031-1033.

[2] Фураев В.З. О разрешимости в целом первой краевой задачи для обобщенного уравнения Буссинеска // Дифференц. уравнения. 1983. Т. 19. №11. С. 2014-2015.

[3] Гладков А. Л. Задача Коши в классах растущих функций для некоторых нелинейных псевдопараболических уравнений // Дифференц. уравнения. 1988. Т. 24. № 2. С. 277-288.

[4] Кожанов А. И. Положительность решений нелинейных псевдопараболических уравнений // Дифференциальные уравнения с частными производными. Сб. научн. тр. Новосибирск: ИМ CO AH CCCP, 1989. C. 79-92.

[5] Кожанов А.И. Теоремы сравнения и разрешимость краевых задач для некоторых классов эволюционных уравнений типа псевдопараболических и псевдогиперболических. Препринт № 17. Новосибирск: ИМ СО АН СССР, 1990.

[6] Кожанов А. И. Параболические уравнения с нелинейным нелокальным источником // Сиб. матем. ж. 1994. Т. 35. № 5. С. 1062-1073.

[7] Самарский А. А., Галактионов В.А., Курдюмов С. П., Михайлов А. П. Режимы с обострением в задачах для квазилинейных параболических уравнений. М.: Наука, 1987.

[8] Лионс ЖК.-Л. Некоторые методы решения нелинейных краевых задач. М.: Мир, 1972.

[9] Кожанов А. И., Л арькин Н. А., Яненко Н. Н. Смешанная задача для некоторых классов уравнений третьего порядка. Препринт. Новосибирск: ИТПМ СО АН СССР, 1980.

Институт математики СО РАН, г. Новосибирск 